\title{
O impacto da saúde bucal nas atividades diárias de indivíduos de 50 a 74 anos em três distritos sanitários de Porto Alegre/RS
}

\section{Oral health impacts on daily activities of individuals aged 50-74 in three health districts of Porto Alegre/RS}

Vanessa Bittencourt

Claides Abegg**

Victor Nascimento Fontanive F** $^{*}$

\section{Resumo}

Objetivo: investigar a prevalência de problemas bucais e seu impacto nas atividades diárias, bem como sua relação com variáveis sociais e demográficas. Métodos: estudo transversal de base populacional avaliando indivíduos entre 50 e 74 anos $(n=720)$ residentes em três distritos sanitários de Porto Alegre/RS. Utilizou-se uma amostragem por múltiplos estágios. Os dados foram coletados nos domicílios, utilizando-se um questionário contendo aspectos sociais (educação, renda), demográficos (idade, sexo) e o indicador sociodental Oral Impact on Daily Performances (OIDP). Utilizou-se estatística descritiva. Para a análise bivariada entre as variáveis sociodemográficas e o impacto odontológico, foi utilizado o teste qui-quadrado de Pearson. Resultados: mais da metade dos sujeitos $(57,8 \%)$ relataram pelo menos uma atividade diária afetada por problemas bucais. Comer (36,8\%), sorrir (30,7\%) e falar claramente $(19,6 \%)$ foram as atividades diárias com maior frequência de impacto. Não se observou associação significativa entre o impacto e as variáveis sociodemográficas $(p>0,05)$. Os principais problemas bucais causadores de impactos foram: dentadura mal ajustada e perda de dentes. Conclusão: a alta prevalência de impacto encontrada demonstra a influência dos problemas bucais nas atividades diárias dos indivíduos.

Palavras-chave: Qualidade de vida. Saúde bucal. Prevalência. Idoso.

\section{Introdução}

O aumento da expectativa de vida ocorrido nos países desenvolvidos e em alguns países em desenvolvimento como o Brasil teve, entre suas consequências, a mudança do perfil de morbidade, levando à elevação da prevalência das doenças crônicas, incluindo os problemas bucais ${ }^{1}$. Essa mudança implica a reorganização das políticas de atenção à saúde com o intuito de desenvolver ações que favoreçam o bem-estar dos indivíduos considerados de meia-idade (entre 40 e 60 anos) e idosos (60 anos ou mais $)^{1}$.

Portanto, consciente de seu papel fundamental em busca da promoção de saúde é que, recentemente, a comunidade científica na área odontológica tem dedicado maior interesse a questionamentos sobre como os problemas bucais afetam a qualidade de vida dos indivíduos ${ }^{2}$.

Nos últimos anos, foram desenvolvidos vários instrumentos com o objetivo de analisar os impactos sociais, psicológicos, culturais e econômicos provocados pelos problemas bucais. Além disso, esses indicadores possibilitam avaliar a percepção subjetiva dos indivíduos sobre sua saúde e suas necessidades em saúde ${ }^{2}$.

Esses instrumentos têm sido, genericamente, denominados de indicadores sociodentais, sócio-

Mestra em Saúde Bucal Coletiva, Departamento de Odontologia Preventiva e Social, Universidade Federal do Rio Grande do Sul, Porto Alegre, RS, Brasil.

Doutora em Epidemiologia, professora do Programa de Pós-Graduação em Odontologia, Universidade Federal do Rio Grande do Sul, Porto Alegre, RS, Brasil.

**** Mestre em Saúde Bucal Coletiva - Serviço de Saúde Comunitária, Grupo Hospitalar Conceição, Porto Alegre, RS, Brasil. 
-odontológicos ou indicadores de qualidade de vida relacionados à saúde bucal e contribuem para que se estabeleça um diagnóstico mais amplo das necessidades em saúde a partir da percepção dos sujeitos. As avaliações de necessidade em saúde baseadas exclusivamente em critérios clínicos refletem somente um aspecto da condição de saúde bucal e podem ter pouca relação com a percepção subjetiva dos indivíduos sobre a saúde bucal. Desse modo, não permitem a determinação do real impacto dos problemas bucais na vida diária dos indivíduos ${ }^{3}$.

Os indicadores sociodentais podem ser usados como um complemento da avaliação das informações clínicas e possibilitam identificar pessoas ou populações que necessitam de ações curativas, preventivas ou educativas, contribuindo, assim, para o planejamento de políticas e serviços voltados às reais necessidades da população $0^{4}$.

Entre os indicadores sociodentais, o Oral Impact of Daily Performances, (OIDP), desenvolvido por Adulyanon e Sheiham ${ }^{2}$ (1997), é considerado um dos mais completos, por quantificar o impacto odontológico nas atividades diárias dos indivíduos, por meio da frequência e severidade. Esse indicador também procura mensurar a percepção do indivíduo sobre esse impacto, bem como avaliar suas causas, podendo ser usado no planejamento e na avaliação de programas de saúde ${ }^{2}$.

Considerando o caráter recente do desenvolvimento de instrumentos de avaliação das repercussões dos problemas bucais na vida diária dos indivíduos, bem como a escassez de pesquisas sobre o tema em indivíduos de meia-idade e idosos no Brasil ${ }^{4,5}$, justifica-se a realização de estudos que avaliem com maior profundidade esse tema. Assim, o objetivo principal deste estudo foi investigar a prevalência do impacto da condição de saúde bucal nas atividades da vida diária e sua relação com variáveis sociodemográficas de indivíduos com 50 a 74 anos residentes em Porto Alegre/RS.

\section{Métodos}

Este é um estudo transversal de base populacional realizado em Porto Alegre/RS entre maio de 2008 e março de 2009. A cidade de Porto Alegre é constituída por 82 bairros, agrupados em 16 distritos sanitários. Foram selecionados, aleatoriamente, três distritos: Partenon, Glória e Leste. A população alvo foi composta por indivíduos entre 50 e 74 anos. Desenvolveu-se um estudo piloto, antes da pesquisa principal, envolvendo 40 usuários do Centro de Saúde - Escola Murialdo em Porto Alegre, com o objetivo de avaliar a adequação dos instrumentos da pesquisa e definir aspectos operacionais do estudo.

O cálculo amostral foi realizado projetando-se uma prevalência para o impacto odontológico de $50 \%$, um intervalo de confiança de 95\% (IC 95\%) e margem de erro bilateral de 4 pontos. Obteve-se uma amostra de 601 sujeitos. Considerando-se a possibilidade de perdas, calculou-se um acréscimo de cerca de $10 \%$, chegando-se a 661 indivíduos. Por tratar-se de amostragem por múltiplos estágios, foi considerado um efeito de delineamento de 1.2, obtendo-se um n final correspondente a 793 sujeitos.

Utilizou-se amostragem aleatória proporcional de múltiplos estágios. No primeiro estágio, foram sorteados, de forma aleatória simples, três distritos sanitários. No segundo, foram sorteados, aleatoriamente, os setores censitários com base em listagens e mapas obtidos na Secretaria Municipal de Saúde (SMS) de Porto Alegre. No terceiro estágio, foram sorteados os quarteirões. A seguir, sorteou-se a esquina e o domicílio correspondentes ao ponto de início da coleta de dados. O número de indivíduos em cada distrito sanitário foi alocado proporcionalmente ao total de pessoas na faixa etária de 50 a 74 anos, residentes nos respectivos distritos. Em cada domicílio, foi realizada somente uma entrevista. Foram excluídas pessoas visitantes não residentes no domicílio, domicílios desabitados, asilos e casas comerciais.

Os dados foram coletados por intermédio de um questionário composto pelo OIDP e questões relacionadas a dados sociais e demográficos. Essas questões foram adaptadas do estudo SB Brasil ${ }^{6}$.

Utilizou-se a versão modificada do OIDP proposta por Sheiham et al. ${ }^{7}$ (2001); Tsakos et al. ${ }^{8}$ (2001). Esse instrumento é composto por 11 performances ou atividades cotidianas, envolvendo as dimensões física, psicológica e social: comer; falar claramente; limpar seus dentes; realizar atividades físicas leves; sair; dormir; sorrir, dar risada e mostrar os dentes sem ficar envergonhado; estado emocional; trabalhar; aproveitar o convívio das pessoas e descansar.

O sistema de classificação para o OIDP quantifica o impacto através da multiplicação da frequência (distribuída em uma escala que varia de 1 a 5) pela severidade (distribuída em uma escala com variação de 0 a 5) para cada uma das 11 atividades diárias. Já o índice individual resulta da soma dos valores dessas 11 atividades diárias. A relação causal dos impactos é estabelecida mediante perguntas feitas aos entrevistados sobre os sintomas percebidos e os prejuízos de todos os impactos sobre suas atividades diárias. Por meio desses questionamentos, estabelece-se maior robustez ao OIDP na avaliação das necessidades de tratamentos específicos. Em etapa anterior, foi realizada a adaptação transcultural do instrumento, incluindo a validação face a face, de conteúdo e de critérios, em uma amostra de conveniência de 200 sujeitos. A confiabilidade interna do OIDP foi de 0,69 (alpha de Cronbach). No teste-reteste, a confiabilidade medida pelo coeficiente intraclasse (ICC) ficou em 0,69.

Os dados foram coletados por quatro entrevistadores e quatro anotadores treinados previamente. A confiabilidade dos dados foi realizada por intermédio da re-entrevista de 5\% da amostra após um intervalo de uma semana. O coeficiente intraclasse foi de 0,85 para o questionário e de 0,80 para o OIDP. 
A variável do escore individual do OIDP foi categorizada em indivíduos sem impacto e indivíduos com impacto. A partir das distribuições das frequências, os escores do OIDP foram divididos em tercis. Os indivíduos que apresentaram impacto nas atividades diárias foram classificados em OIDP baixo, OIDP médio ou OIDP alto. As categorias envolvidas no estudo foram: sem impacto, OIDP = 0: código 0; OIDP baixo - de 0,1 a 3,63: código 1; OIDP médio - de 3,64 a 14,54: código 2; OIDP alto - de 14,55 a 83,64: código 3.

As variáveis demográficas e sociais foram: sexo, idade (50 a 59 anos, 60 a 74 anos), escolaridade ( $\leq 5$ anos ou $\geq 6$ anos), renda pessoal ( $\leq 1$ salário mínimo e $>1$ salário mínimo). Os dados coletados foram digitados em um banco de dados criado no programa Epi-info 6 (Centers for Disease Control and Prevention, Atlanta, Georgia, USA) e analisados com base no programa Stata, versão 9.0 (Stata Corp. ${ }^{\circledR}$, College Station, Texas, USA).

A análise estatística compreendeu a análise descritiva das características gerais da amostra mediante apresentação das frequências. Seguiu-se com análises bivariadas entre as variáveis sociodemográficas e o impacto odontológico, utilizando, para tal, o teste qui-quadrado de Pearson. Os resultados foram considerados estatisticamente significantes quando $\mathrm{p}<$ 0,05 e os intervalos de confiança foram de $95 \%$.

Os indivíduos participantes do estudo tiveram, antes do início da entrevista, uma explicação sobre o objetivo da pesquisa, por meio da leitura do termo de consentimento livre e esclarecido, que foi assinado em duas vias, de igual teor, ficando uma em poder da pessoa entrevistada e outra em poder do pesquisador.

$\mathrm{O}$ presente estudo fez parte de uma pesquisa intitulada "Avaliação do impacto odontológico no desempenho diário dos indivíduos de 50 a 74 anos em Porto
Alegre/RS", que obteve financiamento do Edital SUS/ Fapergs/CNPq 08-04, aprovada pelo Comitê de Pesquisa e Ética da Faculdade de Odontologia da UFRGS no dia 24/01/2006, ata 01/06, processo 46/05.

\section{Resultados}

Ao total, 720 indivíduos, entre 50 e 74 anos, participaram do estudo, perfazendo uma taxa de resposta de $91 \%$. Em relação ao sexo, $304(42,2 \%)$ eram do sexo masculino e 416 (57,8\%), do sexo feminino. A distribuição por sexo foi a seguinte: na faixa de 50 a 59 anos, 167 (43,7\%) eram do sexo masculino e 215 (56,3\%), do sexo feminino. Na faixa de 60 a 74 anos, $137(40,5 \%)$ eram homens e $201(59,5 \%)$ eram mulheres.

Dos indivíduos entrevistados, 31,9\% declararam receber entre nenhum tipo de renda e um salário mínimo, e $68,1 \%$ disseram receber renda pessoal superior a 1 salário mínimo. Do total da amostra, a média de anos de estudo foi de 7,95 ( $\mathrm{DP}=4,61)$. A maioria dos participantes, $447(62,1 \%)$, apresentou 6 anos ou mais de estudo.

Do total de entrevistados, $416(57,8 \%)$ relataram pelo menos uma atividade diária afetada por problemas bucais nos últimos seis meses. As atividades diárias que apresentaram maior impacto foram comer $(36,8 \%)$; sorrir, dar risada e mostrar os dentes sem ficar envergonhado $(30,7 \%)$ e falar claramente $(19,6 \%)$. A prevalência de problemas bucais e o impacto nas atividades diárias da amostra podem ser observados na Tabela 1.

A maioria dos impactos odontológicos apresentou alta frequência e alta severidade. A prevalência das atividades afetadas e os escores médios das frequências e severidades dos impactos estão descritos na Tabela 1.

Tabela 1 - Prevalência, frequência e severidade dos impactos bucais nas atividades diárias

\begin{tabular}{|c|c|c|c|c|c|c|}
\hline \multirow{2}{*}{ Atividade diária } & \multicolumn{2}{|c|}{ Pessoas afetadas } & \multicolumn{2}{|c|}{ Frequência (1-5) } & \multicolumn{2}{|c|}{ Severidade (0-5) } \\
\hline & $(n=416)$ & $\mathrm{N} \%$ & Média & Mediana & Média & Mediana \\
\hline 1. Para comer & 265 & 36,8 & 4,51 & 5 & 2,93 & 3 \\
\hline 2. Para falar claramente & 141 & 19,6 & 4,55 & 5 & 2,84 & 3 \\
\hline 3. Para limpar seus dentes (dentaduras) & 92 & 12,8 & 4,44 & 5 & 2,86 & 3 \\
\hline $\begin{array}{l}\text { 4. Para realizar atividades físicas leves, } \\
\text { como trabalhos domésticos }\end{array}$ & 11 & 1,5 & 3,52 & 4,27 & 3,24 & 4 \\
\hline $\begin{array}{l}\text { 5. Para sair (ex. ir às compras ou visitar } \\
\text { alguém) }\end{array}$ & 61 & 8,5 & 4,05 & 5 & 3,56 & 4 \\
\hline 6. Para dormir & 66 & 9,2 & 4,28 & 5 & 2,81 & 3 \\
\hline $\begin{array}{l}\text { 7. Para sorrir, dar risadas e mostrar os } \\
\text { dentes sem ficar envergonhado }\end{array}$ & 221 & 30,7 & 4,50 & 5 & 3,19 & 4 \\
\hline $\begin{array}{l}\text { 8. Com seu estado emocional, por } \\
\text { exemplo, incomodar-se mais que o } \\
\text { normal }\end{array}$ & 99 & 13,7 & 4,53 & 5 & 3,30 & 4 \\
\hline 9. Para trabalhar & 27 & 3,7 & 4,55 & 5 & 3,29 & 4 \\
\hline $\begin{array}{l}\text { 10. Em aproveitar o convívio com as } \\
\text { pessoas, tais como parentes, amigos } \\
\text { ou vizinhos }\end{array}$ & 86 & 11,9 & 4,65 & 5 & 3,37 & 4 \\
\hline 11. Para descansar & 28 & 3,9 & 4,05 & 5 & 2,91 & 3 \\
\hline
\end{tabular}


Os indivíduos que apresentaram pelo menos uma atividade diária afetada relataram nas entrevistas o principal problema odontológico relacionado à atividade diária afetada. Observou-se que os principais problemas odontológicos mencionados foram: dentadura mal ajustada e perda de dentes.

Não foi observada associação estatística significativa entre as variáveis sociais e demográficas com as atividades diárias afetadas $(p>0,05)$. A variável idade foi a única que apresentou associação estatisticamente significativa com limpar seus dentes ou dentaduras ( $\mathrm{p}=0,012)$, constatando-se que os indivíduos com idade entre 50 e 59 anos apresentaram maior impacto para essa atividade. Os resultados estão apresentados na Tabela 2.

Tabela 2 - Relação de associação entre atividades diárias e variáveis sociodemográficas. Frequências dos indivíduos que relataram impacto

\begin{tabular}{|c|c|c|c|c|c|c|c|c|}
\hline \multirow{2}{*}{$\begin{array}{l}\text { Atividades } \\
\text { diárias }\end{array}$} & \multicolumn{2}{|c|}{ Idade n (\%) } & \multicolumn{2}{|c|}{ Sexo n $(\%)$} & \multicolumn{2}{|c|}{ Escolaridade n (\%) } & \multicolumn{2}{|c|}{ Renda n (\%) } \\
\hline & $\begin{array}{l}50-59 \text { anos } \\
60-74 \text { anos }\end{array}$ & valor $\mathrm{p}^{*}$ & $\begin{array}{l}\text { Fem. } \\
\text { Masc. }\end{array}$ & valor $\mathrm{p}^{*}$ & $\begin{array}{l}\leq 5 \text { anos } \\
\geq 6 \text { anos }\end{array}$ & valor $\mathrm{p}^{*}$ & $\begin{array}{l}\leq 1 \text { salário } \\
>1 \text { salário }\end{array}$ & valor $\mathrm{p}^{*}$ \\
\hline 1. Para comer & $\begin{array}{l}147(55,5) \\
118(44,5)\end{array}$ & 0,321 & $\begin{array}{l}160(60,4) \\
105(39,6)\end{array}$ & 0,296 & $\begin{array}{l}105(39,6) \\
160(60,4)\end{array}$ & 0,485 & $\begin{array}{c}86(32,5) \\
179(67,5)\end{array}$ & 0,823 \\
\hline 2. Para falar claramente & $\begin{array}{l}74(52,5) \\
67(47,5)\end{array}$ & 0,879 & $\begin{array}{l}86(61,0) \\
55(39,0)\end{array}$ & 0,409 & $\begin{array}{l}53(37,6) \\
88(62,4)\end{array}$ & 0,947 & $\begin{array}{c}39(27,7) \\
102(72,3)\end{array}$ & 0,224 \\
\hline $\begin{array}{l}\text { 3. Para limpar seus dentes } \\
\text { (dentaduras) }\end{array}$ & $\begin{array}{l}60(65,2) \\
32(34,8)\end{array}$ & 0,012 & $\begin{array}{l}55(59,8) \\
37(40,2)\end{array}$ & 0,698 & $\begin{array}{l}42(45,7) \\
50(54,3)\end{array}$ & 0,102 & $\begin{array}{l}31(33,7) \\
61(66,3)\end{array}$ & 0,684 \\
\hline $\begin{array}{l}\text { 4. Para realizar atividades } \\
\text { físicas leves, como } \\
\text { trabalhos domésticos }\end{array}$ & $\begin{array}{l}9(81,8) \\
2(18,2)\end{array}$ & 0,055 & $\begin{array}{l}7(63,6) \\
4(36,4)\end{array}$ & 0,699 & $\begin{array}{l}6(54,5) \\
5(45,5)\end{array}$ & 0,252 & $\begin{array}{l}3(27,3) \\
8(72,7)\end{array}$ & 0,738 \\
\hline $\begin{array}{l}\text { 5. Para sair (ex. ir às } \\
\text { compras ou visitar } \\
\text { alguém) }\end{array}$ & $\begin{array}{l}36(58,1) \\
26(41,9)\end{array}$ & 0,408 & $\begin{array}{l}35(57,4) \\
26(42,6)\end{array}$ & 0,937 & $\begin{array}{l}22(36,1) \\
39(63,9)\end{array}$ & 0,755 & $\begin{array}{l}18(29,5) \\
43(70,5)\end{array}$ & 0,670 \\
\hline 6. Para dormir & $\begin{array}{l}34(51,5) \\
32(48,5)\end{array}$ & 0,792 & $\begin{array}{l}34(50,7) \\
33(49,3)\end{array}$ & 0,217 & $\begin{array}{l}23(34,8) \\
43(65,2)\end{array}$ & 0,584 & $\begin{array}{l}17(25,8) \\
49(74,2)\end{array}$ & 0,265 \\
\hline $\begin{array}{l}\text { 7. Para sorrir, dar risadas e } \\
\text { mostrar os dentes sem } \\
\text { ficar envergonhado }\end{array}$ & $\begin{array}{l}115(52,0) \\
106(48,0)\end{array}$ & 0,715 & $\begin{array}{c}134(60,9) \\
86(39,1)\end{array}$ & 0,271 & $\begin{array}{c}83(37,7) \\
137(62,3)\end{array}$ & 0,929 & $\begin{array}{c}71(32,1) \\
150(67,9)\end{array}$ & 0,944 \\
\hline $\begin{array}{l}\text { 8. Com seu estado emo- } \\
\text { cional, por exemplo, } \\
\text { incomodar-se mais que } \\
\text { o normal }\end{array}$ & $\begin{array}{l}57(57,6) \\
42(42,4)\end{array}$ & 0,332 & $\begin{array}{l}59(60,2) \\
30(39,8)\end{array}$ & 0,613 & $\begin{array}{l}35(35,4) \\
64(64,6)\end{array}$ & 0,571 & $\begin{array}{c}26(26,3) \\
73(72,7)\end{array}$ & 0,199 \\
\hline 9. Para trabalhar & $\begin{array}{l}16(59,3) \\
11(40,7)\end{array}$ & 0,510 & $\begin{array}{l}18(66,7) \\
9(33,3)\end{array}$ & 0,340 & $\begin{array}{c}8(29,6) \\
19(70,4)\end{array}$ & 0,366 & $\begin{array}{c}4(14,8) \\
23(85,2)\end{array}$ & 0,052 \\
\hline $\begin{array}{l}\text { 10. Em aproveitar o } \\
\text { convívio com as } \\
\text { pessoas, tais como } \\
\text { parentes, amigos ou } \\
\text { vizinhos }\end{array}$ & $\begin{array}{l}45(52,3) \\
41(47,7)\end{array}$ & 0,885 & $\begin{array}{l}50(58,9) \\
35(41,2)\end{array}$ & 0,848 & $\begin{array}{l}35(40,7) \\
51(59,3)\end{array}$ & 0,571 & $\begin{array}{l}25(29,1) \\
61(70,9)\end{array}$ & 0,548 \\
\hline 11. Para descansar & $\begin{array}{l}16(57,1) \\
12(42,9)\end{array}$ & 0,658 & $\begin{array}{l}18(64,3) \\
10(35,7)\end{array}$ & 0,486 & $\begin{array}{l}11(39,3) \\
17(60,7)\end{array}$ & 0,879 & $\begin{array}{c}6(21,4) \\
22(78,6)\end{array}$ & 0,223 \\
\hline
\end{tabular}

${ }^{*} \mathrm{p}$ valor $=$ teste qui-quadrado de Pearson

Entre os 720 indivíduos entrevistados, a variação do escore individual do OIDP foi de 0 (Escore mínimo) a 83,64 (escore máximo). Também, observou-se que $47,6 \%$ dos indivíduos entrevistados apresentaram OIDP $=0 ; 16,1 \%$, OIDP baixo; $17,0 \%$, OIDP médio e 19,4\%, OIDP alto. 
Tabela 3 - Distribuição dos escores de OIDP, classificados em níveis de impacto, por idade, sexo, escolaridade e renda

\begin{tabular}{|c|c|c|c|c|c|c|}
\hline Variáveis & OIDP $=0 \mathrm{n}(\%)$ & $\begin{array}{c}\text { OIDP baixo } \\
(0,1 \text { a } 3,63) n(\%)\end{array}$ & $\begin{array}{c}\text { OIDP médio } \\
(3,64 \text { a } 14,54) \mathrm{n}(\%)\end{array}$ & $\begin{array}{c}\text { OIDP alto } \\
(14,55 \text { a } 83,64) n(\%)\end{array}$ & $\begin{array}{c}\text { Total } n \\
(\%)\end{array}$ & Valor $\mathrm{p}^{*}$ \\
\hline $\begin{array}{l}\text { Idade } \\
50-59 \text { anos } \\
60-74 \text { anos }\end{array}$ & $\begin{array}{l}176(46,0 \%) \\
166(49,1 \%)\end{array}$ & $\begin{array}{l}57(14,9 \%) \\
59(17,5 \%)\end{array}$ & $\begin{array}{l}66(17,2 \%) \\
57(16,9 \%)\end{array}$ & $\begin{array}{l}84(21,9 \%) \\
56(16,6 \%)\end{array}$ & $\begin{array}{l}383(100 \%) \\
338(100 \%)\end{array}$ & 0,285 \\
\hline $\begin{array}{l}\text { Sexo } \\
\text { Feminino } \\
\text { Masculino }\end{array}$ & $\begin{array}{l}185(44,5 \%) \\
157(51,8 \%)\end{array}$ & $\begin{array}{l}73(17,5 \%) \\
43(14,2 \%)\end{array}$ & $\begin{array}{l}76(18,3 \%) \\
46(15,2 \%)\end{array}$ & $\begin{array}{l}82(19,7 \%) \\
57(18,8 \%)\end{array}$ & $\begin{array}{l}416(100 \%) \\
304(100 \%)\end{array}$ & 0,234 \\
\hline $\begin{array}{l}\text { Escolaridade } \\
\leq 5 \text { anos } \\
\geq 6 \text { anos }\end{array}$ & $\begin{array}{l}129(47,3 \%) \\
214(47,9 \%)\end{array}$ & $\begin{array}{l}42(15,4 \%) \\
74(16,6 \%)\end{array}$ & $\begin{array}{l}51(18,7 \%) \\
71(15,9 \%)\end{array}$ & $\begin{array}{l}51(18,7 \%) \\
88(19,7 \%)\end{array}$ & $\begin{array}{l}273(100 \%) \\
447(100 \%)\end{array}$ & 0,794 \\
\hline $\begin{array}{l}\text { Renda } \\
\leq 1 \text { salário mínimo } \\
>1 \text { salário mínimo }\end{array}$ & $\begin{array}{l}111(48,5 \%) \\
231(47,1 \%)\end{array}$ & $\begin{array}{l}31(13,5 \%) \\
85(17,3 \%)\end{array}$ & $\begin{array}{l}45(19,7 \%) \\
77(17,7 \%)\end{array}$ & $\begin{array}{l}42(18,3 \%) \\
97(19,8 \%)\end{array}$ & $\begin{array}{l}229(100 \%) \\
490(100 \%)\end{array}$ & 0,383 \\
\hline Total & $342(47,6 \%)$ & $116(16,1 \%)$ & $122(17,0 \%)$ & $139(19,4 \%)$ & $720(100 \%)$ & \\
\hline
\end{tabular}

${ }^{*} \mathrm{p}$ valor $=$ teste qui-quadrado de Pearson

A distribuição dos valores de OIDP e sua relação com idade, sexo, escolaridade e renda pessoal, bem como os valores de p estão apresentados na Tabela 3. Não foi encontrada associação significativa ( $p>$ 0,05 ) entre o OIDP e as variáveis sociodemográficas.

\section{Discussão}

Este é o primeiro estudo de base populacional investigando o impacto odontológico nas atividades diárias de indivíduos com idade entre 50 e 74 anos em Porto Alegre/RS. Observou-se que mais da metade dos participantes da pesquisa tiveram impacto em suas atividades diárias devido a problemas relacionados com a boca, dentes ou próteses. Tal resultado é semelhante ao encontrado em outros estudos nacionais e internacionais em indivíduos com faixa etária semelhante ${ }^{9-11}$. A prevalência do impacto tende a ser maior em populações mais jovens, possivelmente devido à influência de sintomas como dor dental/bucal causada por cárie e doença periodontal $^{12,13}$. Autores como Netuveli e Blane ${ }^{14}$ (2008) também discutem o paradoxo do envelhecimento, em que, quanto mais elevada a idade, mais os idosos vão considerando que a vida deve ser desfrutada, sem supervalorizar as perdas e limitações relacionadas com o envelhecimento.

A relação entre a condição de saúde bucal e o bem-estar diário dos indivíduos está bem estabelecida na literatura ${ }^{15,16}$. Da mesma forma, sabe-se que a saúde bucal está associada à saúde geral e que ambas apresentam fatores de risco comuns às doenças crônicas ${ }^{17,18}$.

Os resultados deste estudo indicam que a atividade diária mais afetada por problemas odontológicos foi comer $(36,8 \%)$. Há evidências de que a habilidade de mastigação afeta a escolha dos alimentos da dieta diária dos indivíduos. As perdas dentárias e o uso de próteses mal-adaptadas podem levar à adoção de uma dieta pobre em nutrientes, privi- legiando a ingestão de alimentos menos fibrosos e de consistência mais pastosa, provocando modificações nos hábitos dietéticos e, consequentemente, desequilíbrios nutricionais, bem como problemas de saúde geral ${ }^{18-20}$.

Além de comer, as atividades diárias sorrir e mostrar seus dentes sem ficar envergonhado $(30,7 \%)$ e falar claramente $(19,6 \%)$ foram as que mais impactaram a qualidade de vida relacionada à saúde bucal dos indivíduos. Uma saúde bucal precária pode levar à insatisfação com a aparência e a diminuição da autoestima, trazendo prejuízo nos relacionamentos social, familiar, amoroso e profissional e, em situações extremas, levar ao isolamento e à depressão $0^{19}$.

Os principais problemas causadores de impacto encontrados neste estudo foram dentadura mal ajustada e perda dentária, corroborando resultados encontrados em outros estudos ${ }^{13,20}$. Tal achado pode estar relacionado à falta de atenção à saúde bucal a que os indivíduos foram submetidos ao longo de toda a vida, possivelmente devido à ausência ou dificuldade de acesso a serviços odontológicos. Essa condição de saúde bucal ainda pode ser considerada uma grave consequência do modelo de atenção em saúde bucal do passado, quando predominava um modelo de Odontologia mutiladora ${ }^{21,22}$.

Outra realidade que pode contribuir para tal situação é a carência de serviços de atenção secundária que possam minimizar as sequelas dessa população. Segundo a SMS de Porto Alegre, hoje o município conta com apenas quatro Centros de Especialidades Odontológicas (CEOs), sendo que destes apenas um conta com serviço de reabilitação protética. Além de haver uma demanda expressiva, esse serviço não atende aos distritos que foram avaliados.

Uma alta frequência e severidade de impacto foi observada na população estudada para todas as atividades diárias avaliadas, tendo a frequência mais relatada sido todos os dias ou quase todos os dias, 
mostrando o quanto a saúde bucal interfere na qualidade de vida. Diferentes frequências e severidades foram encontradas por outros autores ${ }^{3,12,23}$.

Adulyanon et al. ${ }^{12}$ (1996), em estudo com uma população tailandesa na faixa de 35 a 44 anos, encontrou um efeito paradoxal em relação à frequência e à severidade dos impactos das diferentes atividades analisadas, ou seja, algumas atividades apresentaram alta frequência e baixa severidade, e vice-versa. Contudo, em um estudo realizado no Brasil, em que a população estudada apresentava a mesma faixa etária, de 35 a 44 anos, foram encontradas alta frequência e alta severidade para quase todas as atividades ${ }^{13}$. É provável que a faixa etária não seja fator predominante na percepção subjetiva dos impactos por parte dos indivíduos, visto que, em uma mesma faixa etária, diferentes frequências e severidades foram encontradas. Assim, mais estudos são necessários para estabelecer a relação do fator idade na frequência e severidade dos impactos. De acordo com a literatura, as diferenças de frequência e severidade do impacto na qualidade de vida relacionadas à saúde bucal podem, em parte, ser atribuídas a aspectos culturais em relação à percepção e manifestação de dor e desconforto ${ }^{16}$.

Diferentemente de outros estudos internacionais $^{16,24}$ e nacionais ${ }^{15,25}$, não foi encontrada, neste estudo, associação estatisticamente significante entre as variáveis sociais de escolaridade e renda pessoal e prevalência do impacto e os escores do OIDP. Uma possível explicação é a homogeneidade na distribuição das variáveis na população estudada, em que não se observou grandes diferenças em relação à renda pessoal e à escolaridade.

Os resultados da associação entre o sexo e o impacto da saúde bucal nas atividades diárias dos indivíduos não são consistentes na literatura. No presente estudo, não se encontrou diferença significativa na distribuição do impacto nas atividades diárias entre os sexos, corroborando os achados de Pereira et al. ${ }^{9}$ (2009) e de Gonçalves et al. ${ }^{26}$ (2004). No entanto, outros estudos encontraram associação entre a variável sexo e o impacto nas atividades diárias ${ }^{13,27}$. As diferenças na percepção do impacto entre os sexos têm sido atribuídas a fatores sociais e normas culturais, incluindo padrões estéticos, em que as mulheres percebem e manifestam mais do que os homens suas necessidades em saúde ${ }^{28}$. Além disso, argumenta-se que condições hormonais e a maior prevalência de doenças sistêmicas podem influenciar a saúde bucal das mulheres, aumentando, assim, a percepção de seu impacto.

Também não se encontrou associação entre sexo, escolaridade, renda e as três atividades diárias mais afetadas, comer (36,8\%); sorrir, dar risadas e mostrar os dentes sem ficar envergonhado (30,7\%) e falar claramente (19,6\%). Em contrapartida, relação estatisticamente significante foi observada entre a variável idade e a atividade diária limpar seus dentes e dentaduras. Os indivíduos da faixa etária entre 50 e 59 anos foram os que apresentaram impacto nessa atividade. Quando se analisou as causas do impacto nas duas faixas etárias estudadas, observou-se que, para os indivíduos mais jovens, a principal causa do impacto para limpar os dentes foi a dor de dente, o que pode explicar a diferença encontrada.

O delineamento adequado, a capacitação dos entrevistadores, o controle de qualidade por intermédio da dupla digitação, o teste-reteste das entrevistas foram estratégias utilizadas para assegurar a validade do estudo, a confiabilidade e a reprodutibilidade dos dados. Entretanto, uma das possíveis limitações do presente estudo é o predomínio de mulheres na amostra. Ao mesmo tempo, não foi encontrada associação estatisticamente significante entre a variável de sexo e a prevalência de impacto nas atividades diárias. Outra limitação do estudo é que seus resultados não podem ser extrapolados para toda a população de Porto Alegre na faixa etária investigada. Mesmo assim, seus principais resultados, mostrando o impacto da condição de saúde bucal na qualidade de vida, são semelhantes aos de outros estudos que incluem adultos e idosos.

A alta prevalência, a severidade e a frequência de impacto relatadas neste estudo sugerem que as políticas de atenção à saúde bucal, no que tange à população dos três distritos sanitários estudados, não têm sido capazes de solucionar problemas que interferem nas atividades diárias de sua população, tais como a perda dentária e o uso de próteses mal-adaptadas. Esses dados salientam a necessidade de serviços odontológicos ofertados a esse grupo populacional objetivando o aumento da sua qualidade de vida relacionada à saúde bucal.

\section{Conclusão}

Tendo como base os resultados obtidos neste estudo, é possível concluir que a prevalência do impacto odontológico nas atividades diárias é alto na população pesquisada, não apresentando, entretanto, associação estatística significativa com as variáveis sociodemográficas incluídas na amostra.

\section{Abstract}

Objective: to investigate the prevalence of oral problems and its impact on daily activities, as well as its relationship with social and demographic variables Methods: cross-sectional population-based study evaluating individuals aged 50 to 74 years old $(n=720)$ from 3 Health Districts in Porto Alegre/RS. A multi-stage sample was used. Data were collected in people's homes, using a questionnaire containing social (education, income) and demographic aspects (age, gender), and the socio-dental indicator Oral Impact on Daily Performances (OIDP). Descriptive analysis was used. For the bivariate analysis among sociodemographic variables and dental care impact, the chi-square and Pearson's 
tests were used. Results: more than half of the subjects $(57.8 \%)$ reported at least one daily activity affected by oral health problems. Eating (36.8\%), smiling (30.7\%), and speaking clearly (19.6\%) were the daily activities with more impact frequency. There was no statistical association between oral impact and sociodemographic variables $(p>0.05)$. The main oral problems causing impacts were: ill-fitting dentures and tooth loss. Conclusion: the high prevalence of oral impact demonstrates the influence of oral problems in the performance of daily activities of the individuals.

Keywords: Quality of life. Oral health. Prevalence. Elderly.

\section{Referências}

1. Seidl EMF, Zannon CML. Qualidade de vida e saúde: aspectos conceituais e metodológicos. Cad Saúde Pública 2004; 20(2):580-8.

2. Adulyanon S, Sheiham A. Oral impacts on daily performances. In: Slade, GD. Measuring oral health and quality of life. Chapell Hill: University of North Carolina; 1997. p. 720.

3. Castro RAL, Portela MC, Leão AT. Adaptação transcultural de índices de qualidade de vida relacionada à saúde bucal. Cad Saúde Pública 2007; 23(10):2275-84.

4. Silva SRC, Fernandes RAC. Autopercepção das condições de saúde bucal por idosos. Rev Saúde Pública 2001; 35(4):34955.

5. Kida IA, Astrom AN, Strand GV, Masalu JR, Tsakos G. Psychometric properties and the prevalence, intensity and causes of oral impacts on daily performance (OIDP) in a population of older Tanzanians. Health Qual Life Outcomes 2006; $4: 56$.

6. Brasil. Ministério da Saúde. Projeto SB Brasil 2003: condicões de saúde bucal da população brasileira 2002-2003. Brasília: Ministério da Saúde; 2004.

7. Sheiham A, Steele JG, Marcenes W, Tsakos G, Finch S, Walls AW. Prevalence of impacts of dental and oral disorders and their effects on eating among older people; a national survey in Great Britain. Community Dent Oral Epidemiol 2001; 29(3):195-203.

8. Tsakos G, Marcenes W, Sheiham A. Evaluation of a modified version of the índex of Oral Impacts on Daily Performances (OIDP) in elderly populations in two european countries. Gerodontology 2001; 18(2):121-30.

9. Pereira KCR, Lacerda JT, Traebert J. The Oral Impact on daily Performances and self-Reported Quality of life in elderly people in Florianópolis, Brasil. Oral Health Prev Dent $2009 ; 7(2): 163-72$.

10. Srisilapanan $P$, Sheiham A. The prevalence of dental impacts on daily performances in older people in Northern Thailand. Gerodontology 2001; 18(2):102-8.

11. Eric J, Stancic I, Tihacek-Sojic L, Kulic L, Popovac A, Tsakos G. Prevalence, severity, and clinical determinants of oral impacts in older people in Bosnia and Herzegovina. Eur J Oral Sci $2012 ; 120(5): 438-43$.

12. Adulyanon S, Vourapukjaru J, Sheiham A. Oral impacts affecting daily performance in a low dental disease Thai population. Community Dent Oral Epidemiol 1996; 24(6):385-9.

13. Gomes AS, Abegg C. O impacto odontológico no desempenho diário dos trabalhadores do Departamento Municipal de Limpeza Urbana de Porto Alegre, Rio Grande do Sul, Brasil. Cad Saúde Pública 2007; 23(7):1707-14.
14. Netuveli G, Blane D. Quality of life in older ages. Br Med Bull 2008; 85(1):113-26.

15. Leão A, Sheiham A. The development of a socio-dental measure of dental impacts on daily living. Community Dent Health 1996; 13(1):22-6.

16. Sheiham A, Steele JG, Marcenes W, Lowe C, Finch S, Bates CJ, et al. The Relationship among dental status, nutrient intake, and nutritional status in older people. J Dent Res 2001; 80(2):408-15.

17. Steele JG, Ayatollahi SMT, Walls AWG, Murray JJ. Clinical factors related to reported satisfaction with oral function amongst dentate older adults in England. Community Dent Oral Epidemiol 2004; 25(2):143-9.

18. De Marchi RJ, Hugo FN, Hilgert JB, Padilha DM. Association between oral health status and nutritional status in south Brazilian independent-living older people. Nutrition 2008; 24(6):546-53.

19. Shinkai RSA, Del Bel Cury AA. O papel da odontologia na equipe interdisciplinar: contribuindo para a atenção integral ao idoso. Cad Saúde Pública 2000; 16(4):1099-109.

20. Dias-da-costa JS, Galli R, Oliveira EA, Backes V, Vial EA, Canuto R, et al. Prevalência de capacidade mastigatória insatisfatória e fatores associados em idosos brasileiros. Cad Saúde Pública 2010; 26(1):79-88

21. Moreira RS, Nico LS, Tomita NE, Ruiz T. A saúde bucal do idoso brasileiro: revisão sistemática sobre o quadro epidemiológico e acesso aos serviços de saúde bucal. Cad Saúde Pública 2005; 21(6):1665-75.

22. Matos DL, Giatti L, Lima-Costa MF. Fatores sócio-demográficos associados ao uso de serviços odontológicos entre idosos brasileiros: um estudo baseado na Pesquisa Nacional por Amostras de Domicílios. Cad Saúde Pública 2004; 20(5):1290-7.

23. Masalu JR, Astrom AN. Aplicability of an abbreviated version of the Oral Impacts on Daily Performaces (OIDP) scale for use among Tanzanian students. Community Dent Oral Epidemiol 2003; 31(1):7-14

24. Slade GD. Derivation and validation of a short-form oral health impact profile. Community Dent Oral Epidemiol 1997; 25(4):284-90.

25. Biazevic MGH, Michel-Crosato E, Iagher F, Pooter CE, Correa SL, Grasel CE. Impact of oral health on quality of life among the elderly population of Joaçaba, Santa Catarina, Brazil. Bras Oral Res 2004; 18(1):85-91.

26. Gonçalves JR, Wassall T, Vieira S, Ramalho AS, Flório FM. Impacto da saúde bucal na qualidade de vida entre homens e mulheres. RGO 2004; 52(4):240-2.

27. Locker D, Miller Y. Subjectively reported oral health status in an adult population. Community Dent Oral Epidemiol 1994; 22(6):425-30.

28. Kandrack MA, Grant KR, Segall A. Gender differences in health related behaviour: some unanswered questions. Soc Sci Med 1991; 32(5):579-90.

Endereço para correspondência:

Claides Abegg

Faculdade de Odontologia da UFRGS

Ramiro Barcelos, 2492 Bairro Santana

90035-000 Porto Alegre - RS

E-mail: claides.abegg@gmail.com

Fone: (51) 33085010

Recebido: 10/01/2013. Aceito: 03/05/2013. 